Pacific Journal of Mathematic 


\section{THE BRANDT CONDITION AND INVERTIBILITY OF MODULES}

\section{HOWARD GORMAN}

Let $L$ be a finite dimensional, symmetric algebra with 1 over the quotient field $K$ of an infinite domain $R$. When $L$ is quaternion algebra and $R$ is a Prüfer ring, it is known that an $R$-module contained in $L$ is invertible if and only if it satisfies a certain relation between it, its dual and its discriminant [7]. We call a symmetric algebra $L$ with this property a Brandt algebra.

We prove: (i) If $R$ is a Prüfer ring, then $L$ is a Brandt algebra if it is 3-dimensional or if it contains only invertible modules. (ii) If $R$ is a valuation ring and $L$ is a Brandt algebra, then any module or its dual is invertible. (iii) If $R \neq K$, then $L$ is not a Brandt algebra if it is generated over $K$ by a single non-cubic element or if it is a matrix algebra over a symmetric algebra $L_{1}$ with $\left(L_{1}: K\right)>1$.

In the late 1920's, a composition of quaternary quadratic forms with rational coefficients, initially formulated by Brandt [2], was reformulated in terms of the multiplication of modules in quaternion algebras. This reformulation was motivated by, and analogous to, Dedekind's theory for the composition of binary quadratic forms. In the binary case, all the modules involved are automatically invertible. For quaternary algebras, this is not so. Brandt dealt with this problem by restricting consideration to forms having the same discriminant and by imposing certain primitivity conditions.

Kaplansky [7], has generalized Brandt's theory to quaternary, quadratic forms over arbitrary Bezout domains (finitely generated ideals are principal). Through the use of the bilinear form on quaternion algebras, he translated Brandt's restriction on forms into a relation between a module in the algebra, its dual and its discriminant, to which we referred above. We call this relation the Brandt Condition, a module which satisfies it a Brandt module and an algebra in which a module is invertible if and only if it is a Brandt module, a Brandt algebra.

The purpose of this paper is to begin a classification of all Brandt algebras. In addition to the results quoted above, we partially settle the question as to when degenerate quaternion algebras are Brandt algebras, and give an example of a cubic algebra which is not a Brandt algebra. 


\section{Preliminaries}

1. Modules and their duals. The following notation will be standard throughout this paper. $L$ will be a finite dimensional, associative algebra with 1 over the quotient field $K$ of an infinite domain $R$. (The assumption that $R$ is infinite is not really restrictive since, if $R$ is finite then $R=K$ and this case is of no interest.) When we say that $L$ is a symmetric algebra over $K$, we mean that there is defined on $L$ a (bilinear) form, denoted by $f$ unless otherwise stated; that is, there is defined on $L$ a bilinear map

$$
f: L \times L \rightarrow K
$$

which is

(i ) symmetric, i.e., $f(a, b)=f(b, a)$ for all $a, b \in L$,

(ii) nonsingular, i.e., $f(a, b)=0$ for all $b \in L$ implies that $a=0$,

(iii) invariant, i.e., $f(a b, c)=f(a, b c)$ for all $a, b, c \in L$.

We shall consider only $R$-modules which are contained in $L$, finitely generated over $R$ and which span $L$ as vector spaces over $K$. When we speak of a module, we shall automatically mean one of this type.

A module is called a semi-order if it contains 1 and consists entirely of elements integral over $R$. A module which is a ring and which contains $R$ is called an order. We remark that, since orders are finitely generated as $R$-modules, they are semi-orders as well.

Via the form $f$, we can identify $L$ with its dual space by the homomorphism which sends $x$ to $f(x$,$) for each x$ in $L$. Since $f$ is nonsingular, this map is one to one, and so, onto as well.

Let $A$ be a module. We define the dual of $A$, written $A^{\sharp}$, to be the set of elements $x$ in $L$ such that $f(x, A) \subseteq R$. Then $A^{\#}$ is an $R$ module. Since $A$ is finitely generated, some $R$-multiple of any element in $L$ is in $A^{\sharp}$, so, $A^{\sharp}$ spans $L$ over $K$.

If $A$ is a free module with basis $a_{1}, \cdots, a_{n}$ and dual basis $b_{1}, \cdots, b_{n}$, then it is clear that each $b_{i}$ is in $A^{\sharp}$. If $z$ is any element of $A^{\sharp}$, and if $f\left(z, a_{i}\right)=r_{i}$ for each $i$, then the nonsingularity of $f$ implies that $z=\sum_{i=1}^{n} r_{i} b_{i}$, i.e., $b_{1}, \cdots, b_{n}$ is a basis for $A^{\#}$ over $R$. This implies that the dual of a free module is a module in the sense of this paper. Further, the same argument applied to $A^{\sharp}$ shows that $a_{1}, \cdots, a_{n}$ is a

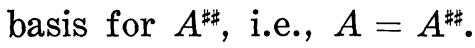

2. The norm and discriminant of a module. For every $x \in L$, we define the norm of $x$, written $N(x)$, to be the determinant of $x$ in the right regular representation of $L$. (We could equally well use the left representation.)

Using the definition of the norm of an element, we define the norm of a module as follows. Suppose that $A$ is a module with ge- 
nerators $a_{1}, \cdots, a_{m}$ over $R$. Let $M_{i}$ be the matrix of $a_{i}$ with respect to some fixed basis of $L$ over $K$. Let $x=\sum_{i=1}^{m} x_{i} a_{i}$ be an arbitrary element of $A$ with each $x_{i} \in R$. Then

$$
N(x)=\operatorname{det}\left(\sum_{i=1}^{m} x_{i} M_{i}\right)=g\left(x_{1}, \cdots, x_{m}\right)
$$

where $g\left(x_{1}, \cdots, x_{m}\right)$ is a homogeneous polynomial of degree $m$ in $x_{1}, \cdots, x_{m}$. We define $N(A)$, the norm of $A$, to be the fractional $R$ ideal generated by the coefficients of $g$.

We claim that $N(A)$ is independent of the generating set chosen. For, let $b_{1}, \cdots, b_{s}$ be another generating set for $A$ and let $b_{i}=\sum_{j=1}^{m} t_{i j} a_{j}$ with each $t_{i j} \in R$. If $z \in A$, then

$$
z=\sum_{i=1}^{s} y_{i} b_{i}=\sum_{\jmath=1}^{m}\left(\sum_{i=1}^{s} t_{i j} y_{i}\right) a_{j},
$$

with each $y_{i} \in R$. Now, substituting $\sum_{i=1}^{s} t_{i j} y_{i}$ for $x_{j}$ in $\mathrm{g}$, for each $j$, we get $N(z)$ on the one hand and a homogeneous polynomial $g_{1}\left(y_{1}, \cdots, y_{s}\right)$ on the other. Since $R$ is infinite, polynomials which are everywhere equal are identical, so $g_{1}$ is the norm polynomial of $A$ with respect to the generators $b_{1}, \cdots, b_{s}$. But the coefficients of $g_{1}$ are $R$-linear combinations of the coefficients of $g$, so the $R$-ideal they generate is contained in $N(A)$. Equality follows by symmetry.

Suppose that $A$ is a free module with basis $a_{1}, \cdots, a_{n}$ over $R$. We define the discriminant of $A$, written $\Delta(A)$, to be the determinant of the matrix $\left(f\left(a_{i}, a_{j}\right)\right)$. It is easy to check that $\Delta(A)$ is unique up to a square of a unit of $R$. If $b_{1}, \cdots, b_{n}$ is a dual basis to $a_{1}, \cdots, a_{n}$, it is not difficult to check that the matrix $\left(f\left(b_{i}, b_{j}\right)\right)$ is the inverse of the matrix $\left(\underline{f}\left(a_{i}, a_{j}\right)\right)$, so that $\Delta\left(A^{\sharp}\right)=(\Delta(A))^{-1}$.

3. The Brandt Condition. We are now in a position to define the Brandt Condition for a free module. We say that the free module $A$ satisfies the Brandt Condition if and only if

$$
N\left(A^{\sharp}\right) \Delta(A) \subseteq N(A) \text {. }
$$

At this point, a little care must be taken. There are, in general, many forms on $L$. If the Brandt Condition were dependent upon the form, a complex situation would arise concerning the definition of Brandt modules and the statement of theorems. The following two lemmas show that the Brandt Condition is independent of the form chosen. The first lemma was proved for algebras with identity by Nakayama [8], and was extended to the infinite dimensional case by Jans [6], again for algebras with 1.

Lemma 1. Let $F$ be any field and $B$ a finite dimensional, as- 
sociative algebra over $F$. Let $f$ be a symmetric non-singular, invariant, bilinear form on $B$. Let $g$ be any symmetric, invariant bilinear form on $B$. Then, there exists $\theta$ in the centroid of $B$ such that $\underline{f}(\theta a, b)=\underline{g}(a, b)$ for all $a, b \in B$. If $B$ contains an identity, $\theta$ is in the center of $B$.

Proof. We identify $B$ with its dual space via $\underline{f}$ as has been already outlined.

Choose any $a \in B$. Then $g(a$,$) is a linear functional on B$ and so, there is $z_{a} \in B$ such that $g(a)=,f\left(z_{a},\right)$. Define the map $\theta: B \rightarrow B$ by $\theta: a \rightarrow z_{a}$. We claim that $\bar{\theta}$ is in the centroid of $B$. It is clearly linear, so we need only show that it commutes with the right and left multiplications of $B$. We give the details for the left multiplications. Let $a, x \in B$. To show that $\theta(a x)=a \theta(x)$, it is enough to show that $f(\theta(a x)-a \theta(x), y)=0$ for every $y \in B$. Now

$$
\underline{f}(\theta(a x), y)=\underline{g}(a x, y)=\underline{g}(a, x y) .
$$

Using the invariance and symmetry of $\underline{f}$ and $\underline{g}$, we find that

$$
\underline{f}(a \theta(x), y)=\underline{f}(y a, \theta(x))=\underline{g}(a, x y) .
$$

Hence $\theta$ is in the centroid.

If $1 \in B$, then $\underline{g}(1)=,\underline{f}(z$,$) for z=\theta(1)$. Then, for any $a, b \in B$, we have that

$$
\underline{g}(a, b)=\underline{g}(1, a b)=\underline{f}(z, a b)=\underline{f}(z a, b) .
$$

Since $\theta$ is in the centroid, $z$ is in the center and the proof is complete.

Since, in the above lemma, $B$ is finite dimensional, $\theta$ will be a unit of the centroid if and only if $\underline{g}$, as well as $\underline{f}$, is nonsingular. For $\theta$ is a nonunit if and only if it annihilates a nonzero $x$ in $B$. But this means that $g(x$,$) is identically zero, i.e., \underline{g}$ is singular. That $\theta^{-1}$ is in the centroid follows without difficulty. Therefore, if $g()=$, $\underline{f}\left(\theta()\right.$, ), we have that $\underline{f}()=,\underline{g}\left(\theta^{-1}(),\right)$. This makes the following definition symmetrical.

If $B$ is a finite dimensional algebra over a field $F$, and $g$ and $h$ are nonsingular bilinear forms on $B$, we say that $g$ and $\underline{h}$ are equivalent if there is $\theta$ in the centroid of $B$ such that $\underline{g}()=,\underline{h}(\theta()$,$) .$

LEMMA 2. Let $L$ be symmetric under equivalent forms $\underline{f}$ and $g$. Let $A$ be a module. Then $A$ satisfies the Brandt Condition with respect to $\underline{f}$ if and only if it satisfies the Brandt Condition with re- 
spect to $g$.

Proof. Write $\Delta_{f}(A)$ and $A_{f}^{\sharp}$ for the discriminant and dual with respect to $\underline{f}$ and do similarly with respect to $\underline{g}$. Suppose that $\underline{f}(z a, b)=$ $\underline{g}(a, b)$ for all $a, b \in L$. Then, $\Delta_{\underline{g}}(A)=N(z) \Delta_{\underline{f}}(A)$.

Choose $t \in L$. Then $g(t, A) \subset R$ if and only if $\underline{f}(z t, A) \subset R$. Since $z$ is a unit of $L$, this implies that $A_{\underline{g}}^{\sharp}=z^{-1} A_{\underline{f}}^{\sharp}$. So,

$$
N\left(A_{\underline{g}}^{\sharp}\right)=N(z)^{-1} N\left(A_{\underline{f}}^{\sharp}\right) \text {. }
$$

This shows that

$$
N\left(A_{\underline{g}}^{\sharp}\right) \Delta_{\underline{g}}(A)=N\left(A_{\underline{f}}^{\sharp}\right) \Delta_{f}(A) .
$$

Since $N(A)$ is independent of $\underline{f}$ and $\underline{g}$, we have the result.

4. Invertibility of modules. We refer the reader to [4] for full details of the following discussion.

Let $A$ be a module. Then the elements $x \in L$ such that $x A \subseteq A$, and the elements $y \in L$ such that $A y \subseteq A$ each form a ring containing $R$. Further, since $A$ is finitely generated and spans $L$ over $K$, these rings span $L$ over $K$ as well. We call these rings the left and right orders of $A$, respectively, and write $A={ }_{P} A_{Q}$ to indicate that $P$ is the left order of $A$ and that $Q$ is the right order of $A$. When $R$ is a Prüfer ring (nonzero, finitely generated ideals invertible), $P$ and $Q$ are finitely generated and so are orders in the sense of this paper.

We call the set of $x$ in $L$ such that $A x A \subseteq A$ the inverse of $A$, written $A^{-1}$. Again, $A^{-1}$ spans $L$ over $K$ and will be finitely generated when $R$ is a Prüfer ring, so that $A^{-1}$ is a module in this case.

We say that $A$ is left (right) invertible if and only if $A^{-1} A=$ $Q\left(A A^{-1}=P\right)$ and invertible if it is both left and right invertible.

5. Brandt modules and localization. When $R$ is a Prüfer ring, modules are projective since they are both finitely generated and torsion free, and so they are locally free. With this in mind, we define a module to be a Brandt module, for a general $R$, if and only if $A_{M}$ satisfies the Brandt Condition as an $R_{M}$-module for each maximal ideal $M$ of $R$. (The definition only makes sense for modules which are locally free.) We shall call $L$ a Brandt algebra if a module contained in it is invertible if and only if it is a Brandt module.

If $A$ is a free module, we can apply the Brandt Condition to it directly, and it is worth checking that, in this case, $A$ satisfies the Brandt Condition if and only if it does so locally.

Let $a_{1}, \cdots, a_{n}$ be a basis for $A$ over $R$ and let $M$ be a maximal ideal of $R$. Then $a_{1}, \cdots, a_{n}$ is a basis for $A_{M}$ as an $R_{M}$-module, so 
$\Delta(A)=\Delta\left(A_{M}\right)$.

Further, $\left(A^{\sharp}\right)_{M}=\left(A_{M}\right)^{\sharp}$. For, from $\underline{f}\left(A^{\sharp}, A\right) \cong R$, we get immediately that $\left(A^{\sharp}\right)_{M} \subseteq\left(A_{M}\right)^{\sharp}$. Conversely, let $x \in\left(A_{M}\right)^{\sharp}$ and suppose that $f\left(x, a_{i}\right)=r_{i} / s_{i}$ for each $i$ with $r_{i}$ and $s_{i}$ in $R$. Setting $s=\sum_{i=1}^{n} s_{i}$, we see that $s x \in A^{\sharp}$; so $x \in\left(A^{\sharp}\right)_{M}$, and we have equality.

Finally, for any module $B$, the norm polynomial for $B_{M}$ is the same as that for $B$ (use the same basis for $B_{M}$ as for $B$ ) except that the domain has been enlarged to $R_{M}$. So, $N\left(B_{M}\right)=N(B)_{M}$.

Then, the above facts, together with the fact that ideals are equal if and only if they are equal locally, give the result.

Standard arguments show that the concept of invertibility localizes, i.e., $A$ is invertible as an $R$-module if and only if $A_{M}$ is invertible as an $R_{M}$-module for each maximal ideal $M$ of $R$.

The above discussion implies that we need only deal with the local situation in determining the connection between invertibility and the Brandt Condition. In this direction, we note that $R$ is a Prüfer ring if and only if $R_{M}$ is a valuation ring for each maximal ideal $M$ of $R$.

6. Extending the base ring. Suppose that $R$ is a valuation ring with maximal ideal $M$. Then the order of the residue class field $R / M$ plays a significant role in the discussion which follows. We wish to show that we can always assume that $R / M$ is infinite. We follow the technique in [4].

Let $R$ be a valuation ring with maximal ideal $M$. Define $R_{0}=$ $R[x]_{M R[x]}$ where $x$ is an indeterminate which commutes with the elements of $L$. Then $R_{0}$ is the ring of rational functions $h(x) / g(x)$ where $h(x)$ and $g(x)$ are polynomials with coefficients in $R$ and $g(x)$ has at least one coefficient a unit of $R$. Then $R_{0}$ is also a valuation ring (any rational function or its reciprocal is in $R_{0}$ ) and has quotient field $K(x)$. We set $L_{0}=L K(x)$ and, for any module $A$, we let $A_{0}=A R_{0}$. Then $\left(L_{0}: K(x)\right)=(L: K)$ and $A_{0}$ is a finitely generated $R_{0}$-module which spans $L_{0}$ over $K(x)$. Further $R_{0} / M R_{0}$ is infinite. We extend the form $f$ to $L_{0}$ in the obvious way, allowing it to commute with the action of $x$. We have the following

Lemma 3. Let $R$ be a valuation ring. Let $A$ be an $R$-module and let $A_{0}=A R_{0}$. Then $A$ is a Brandt module if and only if $A_{0}$ is a Brandt module.

Proof. Let $a_{1}, \cdots, a_{n}$ be a basis of $A$ over $R$. Then it is also a basis for $A_{0}$ over $R_{0}$. Taking discriminants with respect to this basis, we see that $\Delta(A)=\Delta\left(A_{0}\right)$.

We claim that $\left(A_{0}\right)^{\sharp}=\left(A^{\sharp}\right)_{0}$. For, let $b=\sum_{i=0}^{m} b_{i} x^{i}$ be an arbitrary 
element of $\left(A^{\sharp}\right)_{0}$. (So, each $b_{i}$ is in $A^{\sharp}$.) Then $\underline{f}(b, A) \subseteq R_{0}$, and so, from the bilinearity of $\underline{f}, \underline{f}\left(b, A_{0}\right) \leqq R_{0}$, i.e., $\left(A^{\sharp}\right)_{0} \subseteq\left(A_{0}\right)^{\sharp}$. If

$$
c=\sum_{i=1}^{m} c_{i} x^{i} \in\left(A_{0}\right)^{\sharp}
$$

then $f(c, A) \subseteq R_{0}$, which implies by the transcendency of $x$, that the coefficients of $c$ are in $A^{*}$. Hence, $c \in\left(A^{\sharp}\right)_{0}$, and we have equality.

With respect to the basis $a_{1}, \cdots, a_{n}$, the norm polynomial for $A_{0}$ is the same as that for $A$ except that the domain has been enlarged to $R_{0}$. So, $N\left(A_{0}\right)=N(A) R_{0}$. Similarly, $N\left(A_{0}^{\sharp}\right)=N\left(A^{\sharp}\right) R_{0}$.

If $A$ is a Brandt module, we have that

$$
N\left(A^{\sharp}\right) R_{0} \Delta(A) \subseteq N(A) R_{0},
$$

and the above discussion shows that $A_{0}$ is also a Brandt module. Conversely, if $A_{0}$ is a Brandt module, it satisfies (1) and since $x$ is transcendental, we get that $A$ is a Brandt module by comparing constant terms in (1). The proof is complete.

We remark that the relevent remarks in the above proof show also that if $B$ and $C$ are $R$-modules, then $N(B)=N(C)$ if and only if $N\left(B_{0}\right)=N\left(C_{0}\right)$.

In [4], we proved that a module $A$ is invertible if and only if $A_{0}$ is invertible as an $R_{0}$-module. This fact and Lemma 3 allow us to assume, when discussing invertibility, that when $R$ is a valuation ring with maximal ideal $M$, then $R / M$ is infinite. We do so. This implies, again by [4], that when $R$ is a valuation ring, any module $A$ contains an element of minimal norm (i.e., there is an $x \in A$ such that for all $y \in A, N(x)$ divides $N(y)$ ) and that $B=x^{-1} A$ is a semi-order. (That an element of minimal norm is invertible is also shown in [4].)

We shall need the following lemma.

Lemma 4. Let $R$ be a Prüfer ring and let $A$ be a semiorder. Then $N(A)=R$.

Proof. The hypotheses localize, so we may assume that $R$ is a valuation ring. Let $R_{0}$ be the extension of $R$ previously described and let $A_{0}=A R_{0}$. Let $N_{1}(A)$ be the $R$-ideal generated by the norms of the elements of $A$ and let $N_{1}\left(A_{0}\right)$ be the $R_{0}$-ideal generated by the norms of elements of $A_{0}$. Since $A$ is a semi-order $N_{1}(A)=R$.

Let $z=h(x) / g(x)$ be any element of $A_{0}$ with $h(x)=\sum_{i=0}^{m} a_{i} x^{i}$ with $a_{i} \in A$ for each $i$. Then, a moments reflection shows that the constant term of $N(z)$ is $N\left(a_{0}\right) / g^{n}$ (where $n=(L: K)$ ), i.e., the constant terms of norms of elements of $A_{0}$ are just norms of elements of $A$ times a unit 
of $R_{0}$. Further, $N_{1}(A) \subseteq N_{1}\left(A_{0}\right)$ since $A \subseteq A_{0}$. This means that $N_{1}\left(A_{0}\right) \cap K=N_{1}(A)=R$. In general, we have that $N(A)=N\left(A_{\jmath}\right) \cap K$. But in [4], we showed that the infinity of the residue class field of $R_{0}$ implies that $N\left(A_{0}\right)=N_{1}\left(A_{0}\right)$. So, $N(A)=R$ and we are done.

We remark in passing, that, for any $R$-module $A, N_{1}(A) \subseteq N(A)$. This follows once we observe that the norms of elements of $A$ are linear combinations of the coefficients of the norm polynomial.

We use the extension of the base ring to extract one more fact. Let $A$ be a module and let $u \in L$. We claim that $N(u A)=N(u) N(A)$. Extend the base ring. Then we have, in general, that

$$
N_{1}\left(u A_{0}\right)=N(u) N_{1}\left(A_{0}\right) \text {. }
$$

But now $N$ and $N_{1}$ are the same on modules. So, by replacing $N_{1}$ with $N$ in (2) and intersecting with $K$, we get the result.

\section{The Brandt Condition AND Invertibility}

7. Invertible modules which are Brandt. We say that a module $A={ }_{P} A_{Q}$ is left (right) principal if there is $x \in A$ such that $A=$ $P x(=x Q)$. We remark that since $A$ spans $L$ over $K, x$ is a unit of $L$. Hence $A=x\left(x^{-1} P x\right)$. It is easy to check that $x^{-1} P x=Q$, so that left principal implies right principal. It is clear that principal modules are invertible.

We are going to prove that when $R$ is a Prüfer ring, modules which are locally principal are Brandt. (Such modules are, of course, invertible.) This result is not as special as it may seem, for, there are many algebras which have the property that modules are invertible if and only if they are locally principal. When $R$ is a Prüfer ring, algebras with an involution (see [7]) and algebras which are commutative module their radical (see [4]) have this property. Further, when $R$ is a Dedekind ring and $L$ is either central simple or separable, ideals of maximal orders are locally principal. (see [1] and [3] respectively.) With these facts in mind, we begin the development.

We form pairs $[A, a]$, where $A$ is a free module and $a \in K-\{0\}$. We define the discriminant of the pair $[A, a]$ to be $\Delta(A) / a^{2}$ and its norm to be $N(A) / a$. We say that the pair is primitive if $N(A)=a R$. The theorem will be an easy consequence of the following lemma modified from Theorem 9 of [5].

Lemma 5. Let $R$ be a valuation ring. Let $A, B$ and $C$ be modules with $A$ principal, and suppose that $B C \cong A$. Let the pairs $[B, b],[C, c]$ and $[A, b c]$, with $b$ and $c$ nonzero, have the same discriminant and suppose that $[A, b c]$ is primitive. Then $A$ is a Brandt module. 
Proof. From $f\left(B C, A^{\sharp}\right) \cong R$, it follows by invariance that $f\left(B, C A^{\sharp}\right) \subseteq R$. Hence, $C A^{\sharp} \cong B^{\sharp}$ and so, for any $t \in A^{\sharp}, C t \subseteq B^{\sharp}$. This gives that $\Delta(C t) R \cong \Delta\left(B^{\sharp}\right) R$ which implies that

$$
N(t)^{2} \Delta(C) R \cong \Delta\left(B^{\sharp}\right) R \text {. }
$$

Since $N\left(A^{\ddagger}\right)$ is principal ( $R$ is a valuation ring), (3) implies that

$$
N\left(A^{\ddagger}\right)^{2} \Delta(C) \leqq \Delta\left(B^{\ddagger}\right) R=\Delta(B)^{-1} R .
$$

Since $\Delta(B) / b^{2}=\Delta(C) / c^{2}=\Delta(A) / b^{2} c^{2}$, we get that $\Delta(B)=\Delta(A) / c^{2}$ and $\Delta(C)=\Delta(A) / b^{2}$. Substituting these equalities in (4) and taking square roots gives that

$$
N\left(A^{\sharp}\right) \Delta(A) \subseteq b c R=N(A),
$$

i.e., $A$ is a Brandt module and we are done.

We can now deduce

Theorem 1. Let $R$ be a Prüfer ring with quotient field K. Let $L$ be a finite dimensional, symmetric algebra with 1 over $K$ with the property that invertible modules are locally principal. Then every invertible module is a Brandt module.

Proof. Let $A={ }_{P} A_{Q}$ be an invertible module. We assume that $R$ is a valuation ring; so, $A$ is principal. Then the theorem follows from the lemma by setting $B=P, C=A, b=1$ and $c=N(A)$.

In [4], we classified those algebras $L$ which contain only invertible modules when $R$ is a Prüfer ring. We list them.

LIST A. (1) $K \oplus$ trivial algebra (module sum).

(2) 2-dimensional field extensions of $K$.

(3) $K \oplus K$ (ring sum).

(4) Algebras of generalized $2 \times 2$ triangular matrices, i.e., subalgebras of $m \times m$ matrices the general element of which has the form

$$
\left(\frac{a I_{1}}{0} \mid \frac{{ }^{*}}{b I_{2}}\right)
$$

where $a, b \in K$ and $I_{1}$ and $I_{2}$ are identity matrices.

In each case, these algebras have an involution (and are commutative module their radical); so they will satisfy the hypotheses of Theorem 1 if they are symmetric algebras. We deal with each one separately. 
(i) $K \oplus$ trivial algebra has a nonsingular form if and only if the trivial algebra is zero.

(ii) If $L$ is either a 2-dimensional field over $K$ or $K \oplus K$, choose a basis $1, u$ for $L$ over $K$ with $u^{2}=r u+s, r, s \in K$. Define a bilinear form $\underline{f}$ on this basis by

$$
\underline{f}(1,1)=0 \text { and } \underline{f}(1, u)=1 .
$$

Insist that it be symmetric and extend it to higher powers of $u$ by invariance and reduction. For example, we define

$$
\underline{f}(u, u)=\underline{f}\left(1, u^{2}\right)=\underline{f}(1, r u+s)=r .
$$

For convenience, we shall refer to this method of extension as extension by invariance. Since $L$ is commutative, it involves no contradictions, and gives a nonsingular form. (The discriminant of the form is 1.)

(iii) For generalized $2 \times 2$ triangular matrices, use the reduced trace $\underline{T}$ to define the form, i.e., if $M$ is the matrix appearing in (4), above, then $\underline{T}(M)=a+b$. Then, define the form by $\underline{f}(X, Y)=\underline{T}(X Y)$ for any matrices $X$ and $Y$ in the algebra. This gives a nonsingular form as required.

Now, suppose that $L$ is a symmetric algebra from List $A$. Then, since all modules contained in $L$ are invertible ( $R$ Prüfer), certainly the Brandt modules are. Conversely, $L$ satisfies the hypotheses of Theorem 1, so invertible modules are Brandt. Hence, every symmetric algebra in List $A$ is a Brandt algebra.

8. Three dimensional algebras are Brandt. We are going to show that when $R$ is a Prüfer ring and $L$ is a 3-dimensional algebra with 1 over $K$, then $L$ is a Brandt algebra.

First, we remark that we may assume that $L$ is spanned over $K$ by the powers of a nonquadratic element. For, if this is not the case, then $L$ is quadratic and it follows as a corollary to the work in [5] that $L$ is one of the algebras in List $A$. We assume for the remainder of this section that $L$ is spanned over $K$ by the powers of a nonquadratic element.

We may now state the theorem.

THEOREM 2. Let $R$ be a Prïfer ring with quotient field $K$. Let $L$ be a 3-dimensional algebra with 1 over $K$. Then $L$ is a Brandt algebra.

Proof. We proceed by a number of steps.

(a) We assume that $L=K[u]$, where $u^{3}=a u^{2}+b u+c$, with 
$a, b, c \in R$. Further, we assume that $R$ is a valuation ring with infinite residue class field.

(b) We define a form $\underline{f}$ on the basis of $L$ by

$$
\underline{f}\left(1, u^{2}\right)=1 \text { and } \underline{f}(1, u)=\underline{f}(1,1)=0,
$$

and extend $f$ to all of $L$ by invariance. The discriminant of $\underline{f}$ with respect to this basis is 1 , so $f$ is nonsingular.

(c) Since $L$ is commutative, invertible modules are principal, and so, by Theorem 1, invertible modules are Brandt. The rest of the proof will be devoted to proving the converse.

(d) Let $A$ be a Brandt module. We show that we may assume that $A$ is a semi-order. Let $x \in A$ be an element of minimal norm and let $B$ be the semi-order $x^{-1} A$. It is clear that $B$ is invertible if and only if $A$ is, so we can assume that $A$ is a semi-order to start with if we can show that $B$ is a Brandt module.

Now $\Delta(B)=\Delta\left(x^{-1} A\right)=N(x)^{-2} \Delta(A)$. We know also that $B^{\sharp}=x A^{\sharp}$. For, if $z \in L$, then $\underline{f}(z, A) \subseteq R$ if and only if $\underline{f}(z, x B) \subseteq R$. Using invariance and the fact that $x$ is a unit, we get the result. So, $N\left(B^{\sharp}\right)=$ $N(x) N\left(A^{\sharp}\right)$. Finally, $N(A)=N(x B)=N(x) R$. Then, to show that $B$ is a Brandt module, we need only substitute for $A$ in terms of $B$ in the Brandt relation for $A$.

Therefore, we may assume that $A$ is a semi-order, so that the Brandt Condition on $A$ becomes

$$
N\left(A^{\sharp}\right) \Delta(A) \subseteq R .
$$

To show that $A$ is invertible, it is sufficient (and necessary, see [4]) to show that it is an order. This we do.

(e) If $A$ is a semi-order, it contains $R$ as a pure submodule and therefore, as a direct summand (see [4]), so we can include 1 in a free basis of $A$ over $R$. Let $1, v_{1}, v_{2}$ be such a basis. Since $A$ spans $L$ over $K$ and $R$ is infinite, $A$ contains an infinite number of nonquadratic elements. Then, it is not hard to show that we can assume that $v_{1}$ and $v_{2}$ are, themselves, nonquadratic. Hence $v_{2}$ is a linear combination of $1, v_{1}$ and $v_{1}^{2}$. Since $L=K\left[v_{1}\right]$, we may as well choose $u=$ $v_{1}$. Hence, we may assume that

$$
A=\left(1, u, e+f u+g u^{2}\right) / R,
$$

with $e, f$ and $g$ in $K$.

(f) Using the form defined on $L$, we compute that $\Delta(A)=g^{2}$.

(g) We compute $A^{\sharp}$. If

$$
v=x^{\prime}+y^{\prime} u+z^{\prime} u^{2}
$$


is an arbitrary element of $A^{\sharp}$, then $f(1, v) \in R$ implies that

$$
z^{\prime}=z \in R
$$

$\underline{f}(u, v) \in R$ implies that

$$
y^{\prime}+a z^{\prime}=y \in R
$$

and $\underline{f}\left(e+f u+g u^{2}, v\right) \in R$ implies that

$$
x^{\prime} g+y^{\prime}(f+a g)+z^{\prime}\left(e+a f+\left(a^{2}+b\right) g\right)=x \in R .
$$

Solving these equations for $x^{\prime}, y^{\prime}$ and $z^{\prime}$ in terms of $x, y$ and $z$, and substituting in (5), we find that the general element of $A^{*}$ is of the form

$$
v=g^{-1}(x-(f+a g) y-(e+b g) z)+(y-a z) u+z u^{2},
$$

for arbitrary $x, y$ and $z$ in $R$.

To compute $N\left(A^{\sharp}\right)$, we apply $v$ to the basis $1, u$ and $u^{2}$, and find the determinant polynomial of the matrix thus developed. Below, we list the coefficients which are relevent. $g^{2}$ times the coefficient of

$$
\begin{gathered}
x^{3} \text { is } g^{-1} . \\
y^{3} \text { is }-f^{3} g^{-1}-2 a f^{2}-a^{2} f g+b f g+c g^{2}+a b g^{2} . \\
z^{3} \text { is }-e^{3} g^{-1}-b e^{2}-a c e g+c^{2} g^{2} .
\end{gathered}
$$

We are assuming that these are in $R$ by the Brandt Condition.

Further, since $A$ is a semi-order, $e+f u+g u^{2}$ is integral over $R$. We find that $e+f u+g u^{2}$ satisfies the polynomial

$$
w^{3}-s_{1} w^{2}+s_{2} w-s_{3}=0
$$

where

$$
\begin{aligned}
s_{1}= & a f+a^{2} g+2 b g+3 e, \\
s_{2}= & b^{2} g^{2}-2 a c g^{2}-b f^{2}-a b f g-3 c f g+2 a e f \\
& +2 a^{2} e g+4 b e g+3 e^{2}, \\
s_{3}= & b^{2} e g^{2}-2 a c e g^{2}-b e f^{2}-a b e f g-3 c e f g+a e^{2} f \\
& +a^{2} e^{2} g+2 b e^{2} g+e^{3}-3 a^{2} c f g^{2}+a c f^{2}-b c f g^{2} \\
& +f^{3} c+c^{2} g^{3}+3 a^{2} c f^{2} g .
\end{aligned}
$$

Since $e+f u+g u^{2}$ is nonquadratic, $s_{1}, s_{2}$ and $s_{3}$ are in $R$.

(h) For $A$ to be an order it is necessary and sufficient that $A^{2} \subseteq A$. Now, $A^{2}$ is generated over $R$ by the basis of $A$ together with

$$
u^{2}, u\left(e+f u+g u^{2}\right) \text { and }\left(e+f u+g u^{2}\right)^{2} .
$$


The general element of $A$ has the form

$$
r+e t+(s+f t) u+g t u^{2}
$$

for arbitrary $r, s$ and $t$ in $R$. Then $A$ is an order if elements $r, s$ and $t$ in $R$ can be found so that the elements in (9) have the form of $(10)$.

Reduce the elements in (9) by the minimal polynomial for $u$ and equate them to (10) each in turn. Use the linear independence of $1, u$ and $u^{2}$ to get a system of 3 equations in each case and solve these equations for $r, s$ and $t$. Then we find that

(i) $u^{2} \in A$ if and only if

$$
t=g^{-1}, s=-f g^{-1} \text { and } r=-e g^{-1}
$$

are in $R$;

(ii) $u\left(e+f u+g u^{2}\right)$ is in $A$ if and only if

$$
t=f g^{-1}, s=e+b g-a f-f^{2} g^{-1} \text { and } r=c g-a e-e f g^{-1}
$$

are in $R$; and

(iii) $\left(e+f u+g u^{2}\right)$ is in $A$ if and only if

$$
\begin{aligned}
& t=f^{2} g^{-1}+g\left(a^{2}+b\right)+2 e+2 a f \\
& s=c g^{2}+a b g^{2}+b f g-f^{3} g^{-1}-a^{2} f g-2 a f^{2} \\
& r=a c g^{2}+2 c f g-e f^{2} g^{-1}-b e g-2 a e f-e^{2}-a^{2} e g
\end{aligned}
$$

are in $R$.

(i) Now $g^{-1}$ is in $R$ by the Brandt Condition. Multiply (7) and (8) each by $g^{-2}$. Then the resulting expressions are in $R$ and are seen to be monic polynomials with coefficients in $R$ for $f g^{-1}$ and $e g^{-1}$ respectively. Since $R$ is integrally closed in $K$, we have that $f g^{-1}$ and $e g^{-1}$ are in $R$.

The second element in (iii) is just (7) and so, is in $R$.

A direct calculation shows that $p=f^{2} g^{-1}+a f-b g-e$ satisfies the equation

$$
w^{3}+s_{1} w^{2}+s_{2} w+s_{3} \in R .
$$

The calculation is straightforward, though tedious, with only one point worthy of special mention. The term $f^{6} g^{-3}$ occurs in $p^{3}$. Write

$$
f^{6} g^{-3}=\left(f^{3} g^{-1}\right)^{2} g^{-1} \text {. }
$$

Set (7) equal to some element $q$ in $R$ and square both sides. We note that $\left(f^{3} g^{-1}\right)^{2}$ occurs as one of the terms after squaring. We must substitute for $\left(f^{3} g^{-1}\right)^{2}$ from this squared expression into (12) when we expand (11). By the integral closure of $R$ in $K, p$ is in $R$. 
Since

$$
f^{2} g^{-1}+g\left(a^{2}+b\right)+2 e+2 a f=p+s_{1},
$$

this term is also in $R$.

We have now shown that when the elements of (9) are expressed as $K$-linear combinations of $1, u$ and $e+f u+g u^{2}$, the coefficients of $u$ and $e+f u+g u^{2}$ are, in each case, in $R$. This means that the coefficients of 1 in these expressions together with the basis for $A$ generate $A^{2}$. But $A^{2}$ is again a semi-order since $L$ is commutative, so that these coefficients of 1 , being integral over $R$ and in $K$, are in $R$.

Hence $A^{2} \subseteq A$ and the proof of the theorem is complete.

The following theorem applies to Brandt algebras and generalizes a theorem of Faddeev [3] on 3-dimensional algebras.

THEOREM 3. Let $R$ be a valuation ring. Let $L$ be a symmetric, finite dimensional algebra with 1 over $K$, the quotient field of $R$, with property that Brandt modules are invertible. Then any module or its dual is invertible.

Proof. Let $A$ be a module. The divisibility property of valuation rings implies that either $A$ or $A^{\ddagger}$ is a Brandt module, and hence, invertible by hypothesis.

9. Degenerate quaternion algebras. We call the algebra $L$ with basis $1, u, v, u v$ over $K$ a degenerate quaternion algebra if $u v=-v u$, $u^{2}=0$ and $v^{2}=t \in K$. The theory with regard to these algebras is incomplete; however, the facts which are known are given below. Since we dealt with 3-dimensional algebras in detail, we allow ourselves a more condensed exposition here.

(1) $\operatorname{ch} K \neq 2$. Then, there are no forms. (Singularity is the problem.)

(2) $\operatorname{ch} K=2$. Let $A$ be a semi-order and $R$ a valuation ring. Then, $A$ can be given a basis so that $z=p^{\prime}+q u+r v+s u v$ is the only basis element with nonzero constant term. Since $1 \in A, p^{\prime}=p^{-1}$, with $p \in R$. Since $z$ is integral over $R$,

$$
z^{2}=p^{-2}+r^{2} t \in R \text {. }
$$

In the case where $\left(^{*}\right)$ implies that $p^{-1} \in R$, in particular, when $t=0$, $A$ can always be given a basis of the form

$$
A=(1, a u+b v+c u v, d v+e u v, f u v) / R .
$$


We shall give the salient facts which prove that a Brandt semiorder $A$ with basis as in $\left({ }^{* *}\right)$ is an order. This proves, in particular, that if $t=0$, then $L$ is a Brandt algebra.

The Brandt Condition on $A$ implies that the following expressions are in $R$ :

(i) $a^{-1} b^{2} d^{-1} f k$; $\quad$ (ii) $a^{-1} d f k$;

(iii) $a d f^{-1}+a^{-1} c^{2} d f^{-1} k+a^{-1} b^{2} d^{-1} e^{2} f^{-1} k$.

The fact the basis is integral over $R$ shows that

(iv) $b^{2} k \in R ; \quad$ (v) $d^{2} k \in R$.

The condition that $A^{2} \subseteq A$ requires us to prove that the following expressions are in $R$ :

(a) $b d k ; \quad$ (b) $a^{-1} k(c d+b e) ; \quad$ (c) $a^{-1} b d^{-1} k(c d+b e)$;

(d) $a d f^{-1}+a^{-1} c^{2} d f^{-1} k+a^{-1} b^{2} d^{-1} e^{2} f^{-1} k$;

(e) $a^{-1} b f k ; \quad$ (f) $a^{-1} b^{2} d^{-1} f k$.

We argue as follows. We note that (d) and (f) are just (iii) and (i), respectively. The multiplication of (i) and (ii), and of (iv) and (v), followed by taking square roots in each case, gives that (e) and (a) are in $R$. Finally, we get (b) by multiplying (i) and (ii) and noting that $b k \in R$ (from (iv)); similarly, (c) is obtained by multiplying (i) and (iii) and noting that $b k \in R$. Hence, we are done.

The general case for ch $K=2$ has not yielded to solution. However, we conjecture that, when $R$ is a valuation ring, it also is a Brandt algebra and, further, that any quadratic, symmetric algebra is a Brandt algebra.

Theorem 2 might lead to the conjecture that commutative, cubic algebras are always Brandt. This is not the case. For, let

$$
L=(1, u, v, u v) / K
$$

be commutative with $u^{2}=v^{2}=0$ and ch $K \neq 2$. Then, $L$ is cubic. Let $A$ be the semi-order $\left(^{* *}\right)$. In this case, the Brandt Condition implies only that $a d f^{-1} \in R$. But the demand that $A^{2} \subseteq A$ requires the additional condition that $2 a b f^{-1} \in R$. This need not follow from the Brandt Condition; so, $L$ is not a Brandt algebra.

10. Another norm. There is another norm which can be defined on modules. It has already appeared in $\S 6$ where it was denoted by $N_{1}$. For any module $A$, we defined $N_{1}(A)$ to be the (fractional) $R$-ideal generated by the norms of the elements of $A$. We have already used one of its important properties, namely that if $B$ is a semi-order, then $N_{1}(B)=R$ for any domain $R$. We showed in [4] that when $R$ is a valuation ring with infinite residue class field, then $N_{1}$ and $N$ are the same. It is not difficult to show, in fact, that $N_{1}$ and $N$ will be the same if the residue class field of $R$ is at least as large as $(L: K)$. 
If, however, we wish to define the Brandt Condition using $N_{1}$ instead of $N$, and to examine the connection between this new Brandt Condition and invertibility, we encounter some added difficulties. For, if $R / M$ is too small, $N_{1}$ does not behave well under extension of the base ring, so that we cannot automatically assume that $R / M$ is infinite. Also, in this case, $N_{1}(A)$ need not be finitely generated. (For details, see [4].) The upshot of this is that we cannot assume the existence of an element of minimal norm in $N_{1}\left(A^{\sharp}\right)$ as we did in Theorem 1, and we cannot reduce to the case of a semi-order as we did in Theorem 2. However, Theorems 1 and 2 remain valid for $N_{1}$ as we show below. The validity depends heavily on the assumption that invertible modules are locally principal.

We shall continue to call a module Brandt if it satisfies the Brandt Condition locally with respect to $N$ and shall call it $N_{1}$-Brandt if it satisfies the Brandt Condition locally using $N_{1}$. The validity of Theorems 1 and 2 for $N_{1}$-Brandt modules follows as an easy consequence of Lemmas 6 and 7 respectively.

Lemma 6. Let $R$ be a valuation ring and let $A={ }_{P} A_{Q}$ be a principal module. Then, if $A$ is Brandt, it is $N_{1}$-Brandt.

Proof. Let $A=P x$. Since $R$ is a valuation ring, we have that

$$
N(A)=N(x) R=N_{1}(A) \text {. }
$$

If $A$ is Brandt, we have that

$$
N_{1}\left(A^{\sharp}\right) \Delta(A) \subseteq N\left(A^{\sharp}\right) \Delta(A) \subseteq N(A)=N_{1}(A),
$$

and we are done.

LEMma 7. Let $R$ be a valuation ring and let $A={ }_{P} A_{Q}$ be an $N_{1}$ Brandt module. Let $L$ have the property that any module or its dual is principal. Then $A$ is Brandt.

Proof. We have that

$$
N_{1}\left(A^{\sharp}\right) \Delta(A) \subseteq N_{1}(A) .
$$

If $A$ is not Brandt, it is not principal by Theorem 1 , so $A^{\sharp}$ is principal and therefore, a Brandt module. Then

$$
N(A) \Delta\left(A^{\sharp}\right) \varsubsetneqq N\left(A^{\sharp}\right),
$$

the strict inclusion following from the fact that $A$ is not Brandt. Rearranging (14), we have that

$$
N_{1}(A) \subseteq N(A) \varsubsetneqq \Delta(A) N\left(A^{\sharp}\right)=\Delta(A) N_{1}\left(A^{\sharp}\right),
$$


which contradicts (13) and we are done.

11. Some algebras which are not Brandt. In this section, we prove that some rather elementary algebras are not Brandt algebras.

THEOREM 4. Let $R$ be a domain with quotient field $K$ such that $R \neq K$. Let $L$ be a finite dimensional algebra with 1 over $K$ generated by a non cubic element. Then $L$ is not a Brandt algebra.

Proof. We shall show that $L$ contains a noninvertible Brandt module.

Let $L=K[z]$, with $z$ integral over $R$ and suppose that the minimal polynomial for $z$ is of degree $n$. We define a form $\underline{f}$ on $L$ by

$$
\underline{f}\left(1, z^{n-1}\right)=1 \text { and } \underline{f}\left(1, z^{i}\right)=0, \quad 0 \leqq i<n-1,
$$

and extend $\underline{f}$ to all of $L$ by linearity and invariance. So, $\underline{f}$ is symmetric, invariant and nonsingular. (The discriminant with respect to the basis $1, z, \cdots, z^{n-1}$ is 1 .) We remark that if $L$ were already a symmetric algebra under some form $\underline{g}$, then we can replace $g$ by $\underline{f}$ in what follows since $\underline{f}$ and $g$ would be equivalent.

Let $A$ be the module

$$
A=\left(1, k z, k^{3} z^{2}, k^{3} z^{3}, \cdots, k^{3} z^{n-1}\right) / R,
$$

where $k$ is a nonunit of $R$. We claim that $A$ is Brandt but not invertible.

It is straightforward to check that

$$
\Delta(A) \in k^{6 n-10} R \text {. }
$$

Let $p$ is an arbitrary element of $A^{\sharp}$, written as a $K$-linear combination of the powers of $z$. Forcing $f(p$,$) to send each of the above$ basis elements of $A$ to $R$, and solving the resulting equations, we find that the general element of $A^{\sharp}$ is of the form

$$
x_{0} / k^{3}+\left(x_{1} / k^{3}\right) z+\cdots+\left(x_{n-3} / k^{3}\right) z^{n-3}+\left(x_{n-2} / k\right) z^{n-2}+x_{n-1} z^{n-1} .
$$

Then the determinant of the norm of this element has entries in $k^{-3} R$. This shows that $N\left(A^{\sharp}\right) \subseteq k^{-3 n} R$, and, since $n>3$, we have that

$$
N\left(A^{\sharp}\right) \Delta(A) \subseteq k^{3 n-10} R \varsubsetneqq R \sqsubseteq N(A),
$$

so $A$ is a Brandt module.

But $A$ is not invertible. For, suppose that $q=\sum_{i=0}^{n-1} y_{i} z^{i}$ is an arbitrary element of $A^{-1}$, with each $y_{i} \in K$. Since $1 \in A$, we see that $A^{-1} \cong A$, so that each $y_{i}$, except perhaps $y_{0}$, is in $k R$. From $k z q k z \in A$, 
we get that $y_{0} k^{2}$ is an $R$-multiple of $k^{3}$, so that $y_{0} \in k R$ as well. It follows that if, for $a \in A$ and $b \in A^{-1}$, we express $a b$ as a linear combination of $1, z, \cdots, z^{n-1}$, all the coefficients are in $k R$. So, if $1 \in A A^{-1}$, then $k$ would be a unit of $R$, a contradiction. The proof is complete.

As a corollary, we deduce the next lemma which applies, in particular, to commutative separable algebras.

Lemma 8. Suppose that $R \neq K$, and that $L$ is the product of algebras $L_{1}, \cdots, L_{m}$, such that $(L: K)>3$ and with each $L_{i}$ generated over $K$ by a single element. Then $L$ is not a Brandt algebra.

Proof. Let $L_{i}=K\left[u_{i}\right]$ for each $i$. Let $p_{i}(x)$ be the minimal, monic polynomial for $u_{i}$, and let $p(x)=\prod_{i=1}^{m} p_{i}(x)$.

If $k$ is a nonunit of $R$, all the powers of $k$ are distinct and so, altering the $u_{i}$ by a power of $k$ if necessary, we may assume that if $i \neq j$, then $p_{i}(x)$ and $p_{j}(x)$ have no irreducible factors in common. In particular, $p_{i}\left(u_{j}\right)=0$ if and only if $i=j$.

Let $u=\left(u_{1}, u_{2}, \cdots, u_{m}\right) \in L$. Let $q(x)$ be the minimal, monic polynomial for $u$. Since $p(u)=0, q(x)$ divides $p(x)$. On the other hand, $q(u)=0$ implies that $q\left(u_{i}\right)=0$ for each $i$. So, $p_{i}(x)$ divides $q(x)$ for each $i$. Since distinct $p_{i}(x)$ have distinct irreducible factors, this implies that $q(x)=p(x)$. Since $\operatorname{deg} p(x)=(L: K), L$ is generated over $K$ by $u$. We are done by Theorem 4 .

The fact that the module constructed in Theorem 4 satisfies the Brandt Condition with strict inclusion allows us to deduce the following converse to Theorem 3.

Lemma 9. Let $R$ be a valuation ring with $R \neq K$. Let $L$ be generated over $K$ by a noncubic element. Then $L$ contains a module such that neither it nor its dual is invertible.

Proof. In Theorem 4, the module $A$ is not invertible and satisfies

$$
N\left(A^{\sharp}\right) \Delta(A) \varsubsetneqq N(A) \text {. }
$$

Since $R$ is a valuation ring, (15) shows that $A^{\sharp}$ cannot be Brandt. Since $L$ is commutative, invertible modules are Brandt, so $A^{\sharp}$ is not invertible.

For matrix algebras over symmetric algebras, we have the following

THeorem 5. Let $R$ be a domain with quotient field $K$, such that 
$R \neq K$. Let $L_{1}$ be a finite dimensional, symmetric algebra with 1 over $K$ with $\left(L_{1}: K\right)=m$. Then $L=M_{n}\left(L_{1}\right)$, the $n \times n$ matrices over $L_{1}$, with $n \geqq 2$, is not a Brandt algebra if $n>2$ or $m>1$.

Proof. Let $w_{1}=1, w_{2}, \cdots, w_{m}$ be an integral basis for $L_{1}$ over $K$, and denote the form on $L_{1}$ by $g$. We may assume, further, that the products $w_{i} w_{j}$ are $R$-linear combinations of the basis and that $g\left(w_{i}, w_{j}\right) \in R$ for every $i$ and $j$. Let $e_{i j}$ be the matrix with 1 in the $i$-th row and the $j$-th column and zeros elsewhere. We fix the basis

$$
w_{p} e_{q r}
$$

of $L$ over $K$, with $p=1, \cdots, m$ and $q, r=1, \cdots, n$.

We define the form $f$ on $L$ by

$$
\underline{f}\left(1, w_{p} e_{q r}\right)=\delta_{q r} \underline{g}\left(1, w_{p}\right)
$$

on this fixed basis, where $\delta_{q r}$ is the Kronecker $\delta$. We extend $\underline{f}$ to all of $L$ by invariance (i.e., we define $f(x, y)=\underline{f}(1, x y)$ for all $x, y$ in $L$ ) and linearity. Then $\underline{f}$ is a symmetric, invariant bilinear form. It is also nonsingular. For, suppose that $f(x)=$,0 for some $x \in L$. Write $x=\sum_{r, s, t} a_{r s t} w_{r} e_{s t}$. Then

$$
\begin{aligned}
\underline{f}\left(x, w_{p} e_{u v}\right) & =\sum_{r, s} a_{r s u} \underline{f}\left(1, w_{r} w_{p} e_{s v}\right) \\
& =\sum_{r} a_{r v u} g\left(1, w_{r} w_{p}\right)=g\left(w_{p}, \sum_{r} a_{r v u} w_{r}\right)=0,
\end{aligned}
$$

for every $p, u$ and $v$. Since $g$ is nonsingular, we get that every $a_{r v u}$, and so $x$ also, is zero.

Let $A$ be the module with basis

$$
1, k e_{12}, k e_{21}, k^{3} w_{p} e_{q r},
$$

where the triple $(p, q, r)$ runs over all possible values except $(1,1,2)$, $(1,2,1)$ and $(1,1,1)$, and $k$ is a nonunit of $R$. We shall show that $A$ is a noninvertible Brandt module if $n>2$ or $m>1$.

First, the Brandt Condition. Order the basis of $A$ so that the first four basis elements are $1, k e_{12}, k e_{21}$ and $k^{3} e_{22}$. Suppose that $\left(a_{u v}\right)$ is the symmetric matrix associated with $\Delta(A)$ in this ordering. Then, it is straightforward to see that the $a_{u v}$ have the following properties.

(i ) $a_{11}=n, a_{23}=a_{32}=k^{2}$ and the other $a_{u v}=0$ if $u, v \leqq 3$;

(ii) $a_{1 j} \in k^{3} R$ for all $j>3$;

(iii) $a_{2 j}$ and $a_{3 j}$ are in $k^{4} R$ for all $j>3$;

(iv) $a_{i j} \in k^{6} R$ for $i, j>3$.

Then we need only examine the determinant $\Delta(A)$ to see that 


$$
\Delta(A) \in k^{6 m n^{2}-14} R
$$

If

$$
z=\sum_{r, s, t} x_{r s t} w_{r} e_{s t}
$$

each $x_{r s t} \in R$,

is an arbitrary element of $A^{\sharp}$, we have that $\underline{f}(1, z) \in R$ implies

$$
\sum_{r, s} x_{r s s} \underline{g}\left(1, w_{r}\right) \in R
$$

$\underline{f}\left(k e_{12}, z\right) \in R$ implies

$$
\sum_{r} x_{r 2 \underline{g}} g\left(1, w_{r}\right) \in k^{-1} R
$$

$\underline{f}\left(k e_{21}, z\right) \in R$ implies

$$
\sum_{r} x_{r 12} \underline{g}\left(1, w_{r}\right) \in k^{-1} R
$$

$\underline{f}\left(k^{3} w_{p} e_{i j}, z\right) \in R$ implies

$$
\sum_{r} x_{r j i} \underline{g}\left(w_{p}, w_{r}\right) \in k^{-3} R .
$$

Let $D$ be the determinant of the matrix of this system of equation. Then, we get by Cramer's rule that

$$
x_{r s t}=y_{r s t} / D k^{3} \text {, }
$$

for all $r, s$ and $t$, with $y_{r s t} \in R$. If $D$ is a nonunit of $R$, choose $k=D$. In any case, the result of the above calculations is that

$$
N\left(A^{\sharp}\right) \subseteq k^{-4 m n^{2}} R \text {. }
$$

So, from

$$
N\left(A^{\sharp}\right) \Delta(A) \cong k^{2 m n^{2}-14} R \cong N(A) ，
$$

we see that if $n>2$ or $m>1, A$ is a Brandt module.

But $A$ is not invertible. To see this, let $q \in A^{-1}$. Since $1 \in A$, we get $A^{-1} \subseteq A$; so, $q$ can be writtern as

$$
q=x+\sum_{r, s, t} x_{r s t} w_{r} e_{s t},
$$

for $x, x_{r s t} \in K$ and $(r, s, t) \neq(1,1,1)$, with each $x_{r s t}$ in $k R$. Since

$$
k e_{21} q k e_{12} \in A \text {, }
$$

we get that $x k^{2}$ is an $R$-multiple of $k^{3}$, so $x \in k R$ as well. Then, if we choose $a \in A$ and $b \in A^{-1}$ and express $a b$ (or $b a$ ) as a linear combination of the basis of $A$, we find that the coefficient of 1 is in $k R$. But then, if $1 \in A A^{-1}$ (or $A^{-1} A$ ) $k$ must be a unit of $R$, a contradiction, 
and the proof is complete.

COROLLARY. Let $L_{1}$ be a non-Brandt algebra of the form described in Theorem 4 or 5 . Let $L$ be a product of symmetric algebras one of the factors of which is $L_{1}$. Then $L$ is not a Brandt algebra. (Again, $R \neq K$.)

Proof. Let $A_{1}$ be the module constructed in Theorem 4 or 5 . Choose a module in each other factor of $L$ such that the norm of each module contains $R$. Let $A$ be the product of $A_{1}$ with these other modules.

The Brandt Condition on $A$ reduces to the product of the Brandt Conditions on each of the factors of $A$. Since all of the fractional ideals involved in this product are finitely generated, it follows from the fact that $N\left(A_{1}^{*}\right) \Delta\left(A_{1}\right) \subseteq k R$, for some nonunit $k$ of $R$, that $k$ can be chosen large enough so that

$$
N\left(A^{\sharp}\right) \Delta(A) \subseteq R \cong N(A) .
$$

But $A$ is invertible if and only if each of its factors is. Since $A_{1}$ is not invertible in $L_{1}$, we are done.

We remark finally, that, if $L$ is a 3-dimensional field over $K$, it follows from the fact that $M_{2}(L) \cong M_{2}(K) \bigotimes_{K} L$ is not a Brandt algebra that the tensor product of Brandt algebras need not be a Brandt algebra.

\section{BIBLIOGRAPHY}

1. M. Auslander and O. Goldman, Maximal orders, Trans. Amer. Math. Soc. 97 (1960), $1-24$.

2. H. Brandt, Zur Komposition den Quaternären Quadratische Formen, J. f. Reine und Angewandte Math. 143 (1913), 106-129.

3. D. K. Faddeev, An introduction to the multiplicative theory of modules of integral representations, Trudy Mat. Inst. Steklov 80 (1965) 145-182.

4. H. E. Gorman, Invertibility of modules over Prüfer rings, (to appear in Illinois J. Math.)

5. Thesis, University of Chicago, 1968.

6. J. P. Jans, On Frobenius algebras, Ann. of Math. 69 (1959), 392-407.

7. I. Kaplansky, Submodules of quaternion algebras, Proc. London Math. Soc. 19 (1969), 219-232.

8. T. Nakayama, On Frobenesean algebras II, Ann. of Math. 42 (1941), 1-21.

Received September 26, 1968. This paper was written at the University of Chicago in 1968 with the support of NSF Grant GP-6334.

UNIVERSITY OF CHICAGO 



\section{PACIFIC JOURNAL OF MATHEMATICS}

\section{EDITORS}

\author{
H. SAMELSON \\ Stanford University \\ Stanford, California 94305 \\ RICHARD PIERCE \\ University of Washington \\ Seattle, Washington 98105
}

J. DugundJI

Department of Mathematics University of Southern California Los Angeles, California 90007

BASIL GORDON*

University of California

Los Angeles, California 90024

\section{ASSOCIATE EDITORS}
E. F. BECKENBACH
B. H. NEUMANN
F. WOLE
K. YOSHIDA

\section{SUPPORTING INSTITUTIONS}

\author{
UNIVERSITY OF BRITISH COLUMBIA \\ CALIFORNIA INSTITUTE OF TECHNOLOGY \\ UNIVERSITY OF CALIFORNIA \\ MONTANA STATE UNIVERSITY \\ UNIVERSITY OF NEVADA \\ NEW MEXICO STATE UNIVERSITY \\ OREGON STATE UNIVERSITY \\ UNIVERSITY OF OREGON \\ OSAKA UNIVERSITY \\ UNIVERSITY OF SOUTHERN CALIFORNIA
}

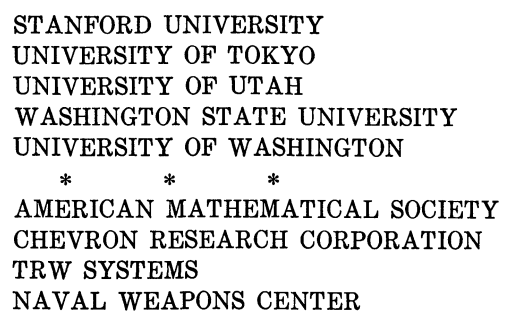

The Supporting Institutions listed above contribute to the cost of publication of this Journal, but they are not owners or publishers and have no responsibility for its content or policies.

Mathematical papers intended for publication in the Pacific Journal of Mathematics should be in typed form or offset-reproduced, (not dittoed), double spaced with large margins. Underline Greek letters in red, German in green, and script in blue. The first paragraph or two must be capable of being used separately as a synopsis of the entire paper. The editorial "we" must not be used in the synopsis, and items of the bibliography should not be cited there unless absolutely necessary, in which case they must be identified by author and Journal, rather than by item number. Manuscripts, in duplicate if possible, may be sent to any one of the four editors. Please classify according to the scheme of Math. Rev. 36, 1539-1546. All other communications to the editors should be addressed to the managing editor, Richard Arens, University of California, Los Angeles, California, 90024.

50 reprints are provided free for each article; additional copies may be obtained at cost in multiples of 50 .

The Pacific Journal of Mathematics is published monthly. Effective with Volume 16 the price per volume (3 numbers) is $\$ 8.00$; single issues, $\$ 3.00$. Special price for current issues to individual faculty members of supporting institutions and to individual members of the American Mathematical Society: $\$ 4.00$ per volume; single issues $\$ 1.50$. Back numbers are available.

Subscriptions, orders for back numbers, and changes of address should be sent to Pacific Journal of Mathematics, 103 Highland Boulevard, Berkeley, California, 94708.

PUBLISHED BY PACIFIC JOURNAL OF MATHEMATICS, A NON-PROFIT CORPORATION

Printed at Kokusai Bunken Insatsusha (International Academic Printing Co., Ltd.), 7-17, Fujimi 2-chome, Chiyoda-ku, Tokyo, Japan.

* Acting Managing Editor. 


\section{Pacific Journal of Mathematics}

\section{Vol. 32, No. $2 \quad$ February, 1970}

Harry P. Allen and Joseph Cooley Ferrar, Jordan algebras and exceptional subalgebras of the exceptional algebra $E_{6} \ldots \ldots \ldots \ldots \ldots \ldots \ldots 283$

David Wilmot Barnette and Branko Grünbaum, Preassigning the shape of a

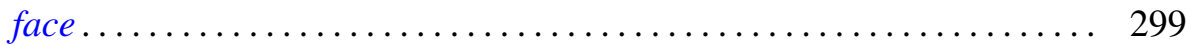

Robert Francis Craggs, Involutions of the 3-sphere which fix 2-spheres . . . . 307

David William Dean, Bor-Luh Lin and Ivan Singer, On k-shrinking and $k$-boundedly complete bases in Banach spaces ................ 323

Martin Engert, Finite dimensional translation invariant subspaces ....... 333

Kenneth Lewis Fields, On the global dimension of residue rings ......... 345

Howard Gorman, The Brandt condition and invertibility of modules ....... 351

Benjamin Rigler Halpern, A characterization of the circle and interval ..... 373

Albert Emerson Hurd, A uniqueness theorem for second order quasilinear

hyperbolic equations ............................... 415

James Frederick Hurley, Composition series in Chevalley algebras ...... 429

Meira Lavie, Disconjugacy of linear differential equations in the complex

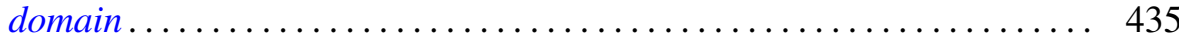

Jimmie Don Lawson, Lattices with no interval homomorphisms ......... 459

Roger McCann, A classification of center-foci ................. 467

Evelyn Rupard McMillan, On continuity conditions for functions . . . . . . . 479

Graciano de Oliveira, A conjecture and some problems on permanents .... 495

David L. Parrott and S. K. Wong, On the Higman-Sims simple group of order $44,352,000$.

Jerome L. Paul, Extending homeomorphisms ................. 517

Thomas Benny Rushing, Unknotting unions of cells .............. 521

Peter Russell, Forms of the affine line and its additive group.......... 527

Niel Shilkret, Non-Archimedean Gelfand theory ................. 541

Alfred Esperanza Tong, Diagonal submatrices of matrix maps.......... 551 